\title{
Aspectos globales de la epidemiología y de la toma de decisiones en la pandemia por COVID-19
}

\author{
Global aspects of epidemiology and decision-making in the COVID-19 \\ pandemic
}

\author{
Vargas-Uricoechea $H^{1}$, Vargas-Sierra $H^{2}$
}

\begin{abstract}
${ }^{1}$ Médico especialista en Medicina Interna y Endocrinología. MSc en Epidemiología. PhDc en Ciencias Biomédicas. Director, Grupo de Investigación en Enfermedades Metabólicas, Departamento de Medicina Interna, Universidad del Cauca, Popayán, Colombia.

${ }^{2}$ Médico, Programa de Posgrado en Medicina Interna, Departamento de Medicina Interna, Facultad Ciencias de la Salud, Universidad del Cauca, Popayán, Colombia.
\end{abstract}

Autor de correspondencia: Hernando Vargas-Uricoechea Correo electrónico: hernandovargasuricoechea@gmail.com

\section{Resumen}

La pandemia actual desencadenada por el SARS-CoV-2/ COVID-19 ha puesto a prueba la capacidad de respuesta de los diferentes sistemas de salud en el mundo. Las pandemias han acompañado al ser humano desde sus orígenes; sin embargo, mucha incertidumbre surge a partir de preguntas acerca de los diferentes escenarios que pueden presentarse durante el curso de la pandemia o posterior a ella. En esta revisión se abordan los aspectos epidemiológicos globales de la COVID-19 y de las probables consecuencias de las diferentes estrategias desde el punto de vista de la salud pública.

Palabras claves: pandemia, SARS-CoV-2, COVID-19, salud pública, epidemiología.

\footnotetext{
Abstract

The current pandemic triggered by the SARS-CoV-2/ COVID-19 has tested the responsiveness of different health systems in the world. Pandemics have accompanied humans since their origins; however, much uncertainty arises from questions about the different scenarios that may arise during or after the pandemic. This review addresses the global epidemiological aspects of COVID-19 and the likely consequences of different strategies from the point of view of public health.

Keywords: pandemic, SARS-CoV-2, COVID-19, public health, epidemiology.
}

\section{Introducción}

En diciembre de 2019, se documentó por parte de la Comisión Municipal de Salud y Sanidad de Wuhan (en la provincia de Hubei, China) una serie de casos de pacientes con infección respiratoria aguda y de etiología desconocida, los cuales tenían como denominador común el haber visitado el mercado local mayorista de Huanan (en Wuhan, China), en donde se comercializaban (entre otras) carnes de todo tipo (pescados y sus derivados, aves de corral, conejos, murciélagos, serpientes y otros animales salvajes) ${ }^{(1)}$. El inicio de los síntomas del primer caso fue el 8 de diciembre de 2019; posteriormente, el 7 de enero de 2020, las autoridades chinas aislaron e identificaron como agente causal a un nuevo virus de la familia Coronaviridae, el cual fue denominado como $S A R S-C o V-2$, y a la enfermedad que produce se le llamó enfermedad por coronavirus (COVID-19). La secuencia genómica del virus fue liberada de forma inmediata a las bases de datos públicas el 10 de enero de 2020 (Wuhan-Hu-1, GenBank Acceso No. MN908947). Para el 12 de enero de 2020, no se habían reportado más casos relacionados con SARS-CoV-2 y se asumieron dos conceptos: el primero, que el centro de propagación había sido el mercado de Huanan (el cual ya había sido clausurado por las autoridades chinas); o que posiblemente los otros casos descritos se habían contagiado en el hospital (infección nosocomial) ${ }^{(2,3)}$. Por ende, se pensó que el virus no era altamente contagioso (ya que no había registro de infección persona-persona). Para ese momento, se concluyó que la transmisión se producía por vías desconocidas durante la estadía hospitalaria; para tal fecha, solo se les había realizado pruebas de laboratorio a los individuos que presentaban síntomas. Sin embargo, diez días después, un total de 571 casos habían sido reportados en 25 diferentes provincias de China, mientras que, en Hubei, ya se habían descrito 17 muertos y 95 pacientes en estado crítico ${ }^{4}$, 5). Con lo anterior, se hizo un estimado de acuerdo con el modelo de enfermedades infectocontagiosas, del Centro de Colaboración (de la Organización mundial de la salud [OMS]); la 
proyección alcanzaba un número de 4000 posibles contagiados y podía llegar a ser cercano a los 10.000. La propagación del virus había sido tal, que el número de pacientes contagiados fue aumentando exponencialmente en la China continental, y para el 30 de enero, se habían reportado 9692 casos en toda China y 90 casos en diferentes países, incluidos Taiwán, Tailandia, Vietnam, Malasia, Nepal, Sri Lanka, Camboya, Japón, Singapur, la República de Corea, Emiratos Árabes Unidos, Estados Unidos de Norte América (EE. UU.), Filipinas, India, Irán, Australia, Canadá, Finlandia, Francia y Alemania ${ }^{(6,7)}$. El primer reporte positivo para las Américas fue el de 19 de enero de 2020 en el estado de Washington (EE. UU.): un hombre de 35 años, con cuadro clínico de tos y fiebre, el cual tenía dentro de sus antecedentes un viaje reciente a Wuhan. El 24 de enero de 2020 se confirma el primer caso de COVID-19 en Europa (Bordeaux, Francia), en una mujer con antecedente reciente también de viaje a China. El 26 de febrero de 2020, el Ministerio de Salud de Brasil reporta el primer caso de COVID-19 en Suramérica, en un hombre de 61 años proveniente de São Paulo, el cual tenía como antecedente un viaje reciente a Europa (Lombardía, Italia). El 6 de marzo de 2020, el Ministerio de Salud y Protección Social de Colombia confirmó el primer caso de SARS-CoV-2 en nuestro país (Bogotá, D.C), una mujer de 19 años, con antecedente de visita reciente a Milán, Italia. El 11 de marzo de 2020, la OMS declaró a la COVID-19 como una pandemia ${ }^{(8,9)}$.

\section{Generalidades del SARS-CoV-2 y de la COVID-19}

Los coronavirus son miembros de la subfamilia Orthocoronavirinae, dentro de la familia Coronaviridae (orden Nidovirales). Esta subfamilia comprende cuatro géneros: Alphacoronavirus, Betacoronavirus, Gammacoronavirus y Deltacoronavirus. El SARS-CoV-2 es un Betacoronavirus (ARN monocatenario de sentido positivo). Hasta ahora, se ha considerado que los reservorios del SARS-CoV-2 son los murciélagos y otros animales sal- vajes; de hecho, se ha identificado que el SARS-CoV-2 tiene un 96,2\% de similitud con un coronavirus del murciélago (denominado BatCoV RaTG13), por lo que se cree que el SARS-CoV-2 se originó a partir de murciélagos infectados ${ }^{(10,11)}$. La COVID-19 es una enfermedad zoonótica; por lo tanto, el SARS-CoV-2 puede transmitirse de los animales a los humanos, pero una vez se produce la infección en el humano, no requiere de los animales para su propagación. Aunque aún no se han identificado los hospederos intermedios, se ha considerado, entre otros, al pangolín (un género de mamíferos folidotos de la familia Manidae). Los coronavirus que afectan al ser humano ( $\mathrm{HCoV}$ ) pueden producir cuadros clínicos que van desde el resfriado común, hasta otros más graves, como los producidos por los virus del síndrome respiratorio agudo grave (SARS) y el síndrome respiratorio de Oriente Próximo (MERS-CoV). El virus se transmite de persona a persona por gotículas, por contacto directo, por fómites y, probablemente, por vía fecal-oral ${ }^{(12,13)}$. El espectro clínico de la COVID-19 se caracteriza por presentar un cuadro clínico muy amplio. Los síntomas más comunes son: fiebre, fatiga, tos seca y producción de esputo. Las manifestaciones, en general, son leves y pueden presentarse de forma progresiva y gradual. Algunos individuos afectados no desarrollan ningún síntoma (por lo que su transmisión puede ser asintomática), y la mayoría (cerca del $80 \%$ ) se recupera de la enfermedad, sin requerir manejo específico; sin embargo, alrededor del $20 \%$ de los afectados desarrolla una enfermedad grave y compleja (neumonía grave, insuficiencia respiratoria, disfunción/falla multiorgánica y muerte). El período de incubación oscila entre 4 y 14 días, pero puede ser de hasta 24 días (0-24 días) ${ }^{(14,15)}$. Por su parte, la tasa de letalidad para la COVID-19 es menor que para el SARS-CoV-2 y que para el MERS, aunque varía de acuerdo con aspectos como la edad y las comorbilidades concomitantes; por ejemplo, dicha tasa es mayor en individuos mayores de 60 años y en aquellos con patologías de base, como diabetes mellitus (DM), hipertensión arterial (HTA), enfermedad pulmonar crónica, cáncer, entre otras (Figura 1) ${ }^{(16,17)}$.

Figura 1. COVID-19: tasas de letalidad en estadios tempranos, según las comorbilidades de base (febrero 2020 ). Fuente: adaptada de la referencia 17.

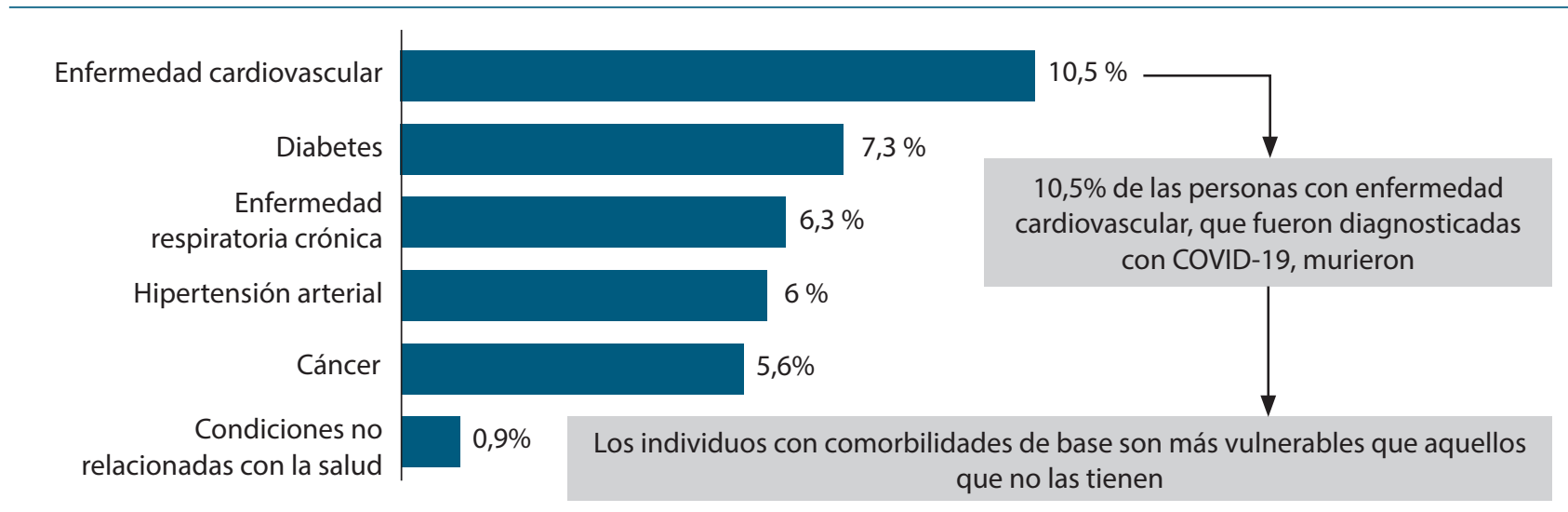




\section{Definición y comportamiento de la pandemia por SARS-CoV-2}

Las pandemias han acompañado al ser humano a lo largo de la historia. Desde la denominada peste antonina, hasta la actual (COVID-19), se han descrito numerosas enfermedades con este carácter, las cuales han tenido como común denominador una alta tasa de letalidad (Tabla 1) ${ }^{(18)}$. En cuanto a los conceptos básicos que deben considerarse en la definición de una pandemia, se destacan:

- Endemia: es la presencia continua de una enfermedad o un agente infeccioso en una zona geográfica determinada. También puede denotar la prevalencia usual de una enfermedad particular en dicha zona. El término hiperendemia significa la presencia constante de una enfermedad con elevada incidencia, y holoendemia, un nivel elevado de prevalencia de la infección a partir de una edad temprana, que afecta a la mayor parte de la población.

- Brote epidémico: se establece cuando existe la aparición de dos o más casos de una misma enfermedad asociados en tiempo, lugar y persona, o también cuando existe un incremento significativo de casos en relación con los valores habitualmente observados, o cuando aparece una enfermedad, condición o riesgo para la salud en una zona hasta entonces libre de ella.
- Epidemia: es la manifestación, en una comunidad o región, de casos de una enfermedad (o un brote) con una frecuencia que exceda netamente de la incidencia normal prevista. El número de casos que indica la existencia de una epidemia varía con el agente infeccioso, el tamaño y las características de la población expuesta, su experiencia previa o falta de exposición a la enfermedad, el sitio y la época del año en que tiene lugar. Por consiguiente, la epidemicidad guarda relación con la frecuencia común de la enfermedad en la misma zona, entre la población especificada y en la misma estación del año. La aparición de un solo caso de una enfermedad transmisible que durante un lapso prolongado no había afectado a una población, o que invade por primera vez una región en la que no había sido diagnosticada anteriormente, requiere la notificación inmediata y una investigación epidemiológica. La presentación de dos casos de una enfermedad de esa naturaleza en los que exista una relación de lugar y tiempo constituye una prueba suficiente de transmisión para que se la considere como epidémica.

- Pandemia: es la afectación de una enfermedad a lo largo de un área geográficamente extensa. Para que una enfermedad adquiera la denominación de pandemia, esta debe tener un alto grado de infectabilidad y un fácil traslado de un sector geográfico a otro ${ }^{(19,20)}$.

Tabla 1. Historia de las pandemias, desde la peste antonina hasta la COVID-19

\begin{tabular}{|c|c|c|c|}
\hline Nombre & Período & Tipo/huésped prehumano & Número de fallecidos (aproximado) \\
\hline $\begin{array}{l}\text { Peste antonina o plaga de } \\
\text { Galeno }\end{array}$ & $165-180$ & Probablemente viruela o sarampión & 5 millones \\
\hline $\begin{array}{l}\text { Epidemia de viruela japonesa } \\
\text { (epidemia de Tenpyō) }\end{array}$ & $735-737$ & Virus variola mayor & 1 millón \\
\hline Plaga de Justiniano & $541-542$ & Bacterias Yersinia pestis/ratas, pulgas & 30-50 millones \\
\hline Peste negra o muerte negra & $1347-1351$ & Bacterias Yersinia pestis/ratas, pulgas & 200 millones \\
\hline $\begin{array}{l}\text { Segunda epidemia mundial de } \\
\text { viruela }\end{array}$ & 1520 & Virus variola mayor & 56 millones \\
\hline Gran plaga o peste de Londres & 1665 & Bacterias Yersinia pestis/ratas, pulgas & 100.000 \\
\hline Gran plaga o peste italiana & $1629-1631$ & Bacterias Yersinia pestis/ratas, pulgas & 1 millón \\
\hline Pandemia de cólera 1-6 & $1817-1923$ & Bacteria Vibrio cholerae & >1 millón \\
\hline $\begin{array}{l}\text { Tercera plaga (tercera pande- } \\
\text { mia de la peste negra) }\end{array}$ & 1885 & Bacterias Yersinia pestis/ratas, pulgas & 12 millones (China e India) \\
\hline Fiebre amarilla & $\begin{array}{l}\text { Finales de } \\
1800\end{array}$ & Virus/mosquitos & 100.000-150.000 (EE.UU.) \\
\hline Gripe rusa & $1889-1890$ & $\begin{array}{l}\text { Virus de la influenza tipo A, subtipo } \\
\text { H3N8 }\end{array}$ & 1 millón \\
\hline Gripe española & 1918-1919 & Virus H1N1/cerdos & 40-50 millones \\
\hline
\end{tabular}


Tabla 1. Historia de las pandemias, desde la peste antonina hasta la COVID-19 (continuación)

\begin{tabular}{l|c|l|c}
\hline \multicolumn{1}{c|}{ Nombre } & \multicolumn{1}{c|}{ Período } & \multicolumn{1}{c}{ Tipo/huésped prehumano } & Número de fallecidos (aproximado) \\
\hline Gripe asiática & $1957-1958$ & Virus influenza A (H2N2) & 1,1 millones \\
\hline Gripe de Hong Kong & $1968-1970$ & Virus influenza (H3N2) & 1 millón \\
\hline VIH/sida & $1981-$ presente & $\begin{array}{l}\text { Virus de la inmunodeficiencia huma- } \\
\text { na /chimpancés }\end{array}$ & $25-35$ millones \\
\hline Gripe porcina (gripe del cerdo) & $2009-2010$ & Virus influenza H1N1/cerdos & 200.000 \\
\hline SARS & $2002-2003$ & Coronavirus/murciélagos, civetas & 774 \\
\hline Ébola & $2014-2016$ & Virus del Ébola/animales salvajes & 11.000 \\
\hline MERS & 2015 & Coronavirus/murciélagos, camellos & 850 \\
\hline SARS-CoV-2/COVID-19 & $2019-p r e s e n t e ~$ & $\begin{array}{l}\text { Coronavirus/murciélagos y } \\
\text { posiblemente los pangolines }\end{array}$ & $>350.000$ (junio de 2020) \\
\hline
\end{tabular}

Como concepto, una enfermedad transmisible es cualquier enfermedad causada por un agente infeccioso específico o sus productos tóxicos, que se manifiesta por la transmisión del mismo agente o sus productos, de una persona o animal infectados o de un reservorio inanimado a un huésped susceptible, en forma directa o indirecta por medio de un huésped intermediario, de naturaleza vegetal o animal, de un vector o del ambiente inanimado ${ }^{(21)}$. El modo en el que pudo transmitirse el SARS-CoV-2 desde la fuente animal a los primeros casos humanos es desconocido. Todo apunta al contacto directo con los animales infectados o sus secreciones. El período de incubación para la COVID-19 (período entre la infección y la manifestación clínica o la aparición de los síntomas) ha variado a medida que ha progresado la investigación; sin embargo, se ha establecido en un período de entre 1 y 14 días (aunque puede ser de 0 a 24 días). Los casos graves generalmente progresan de 7 a 10 días después del inicio de la COVID-19 (22,23). La transmisión de patógenos como el SARS-CoV-2 usualmente se evalúa mediante el número básico de reproducción (R0). La OMS considera que la capacidad de transmisión de este nuevo coronavirus es fuerte, con una transmisibilidad moderada; el R0 puede estar entre 2,0 y 3,0; sin embargo, dichas estimaciones varían, dependiendo del modo del cálculo, el contexto individual y de las medidas poblacionales realizadas o im-

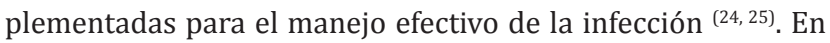
cuanto a los motivos que pueden explicar la alta capacidad de propagación del SARS-CoV-2 están que este sería transmisible durante el período de incubación y que existen casos con sintomatología atípica o individuos asintomáticos, lo que dificulta el diagnóstico. En el análisis de una pandemia, un número importante de definiciones y conceptos deben ser tenidos en cuenta, en especial al hacer los diferentes análisis sobre letalidad, transmisibilidad, período de incubación, R0, entre otros; algunos de estos conceptos se resumen en la Tabla 2.

\section{Epidemiología global y en Colombia de COVID-19 (conceptos básicos)}

\section{Total de muertes confirmadas por COVID-19}

Un punto importante que debe considerarse es que es probable que la cifra total de muertes por COVID-19 sea mayor que la cantidad de muertes confirmadas; esto se debe al número limitado de pruebas y a la atribución de la causa de muerte. La diferencia entre las muertes confirmadas informadas y el total de muertes varía según el país, ya que la forma en que se registran también varía entre las diferentes áreas geográficas (por ejemplo, algunos países solo tienen la capacidad de contar las muertes que se presentan en hospitales, clínicas, etc.), mientras que otros incluyen el número de muertes que ocurren en el hogar; asimismo, la fecha reportada del deceso no necesariamente denota el número de nuevas muertes en ese día; esto se debe al retraso en los informes o reportes de las pruebas ${ }^{(26)}$.

\section{Velocidad a la cual se incrementan las muertes confirmadas por COVID-19}

Los gráficos que simplemente muestran el cambio en las muertes confirmadas a lo largo del tiempo no son muy útiles para responder a la pregunta de cómo se compara la velocidad del brote entre diferentes países. Esto se puede explicar por el hecho de que el brote de COVID-19 no comenzó al mismo tiempo en todos los países. Para esta medición, la trayectoria de cada país comienza el día en que ese país tuvo sus primeras 5 muertes confirmadas. Esto permite comparar qué tan rápido aumentó el número de muertes confirmadas después de que el brote alcanzó una etapa similar en cada país. Ahora bien, observar el número total o diario de muertes confirmadas por COVID-19 no permite comparar la velocidad a la que se aumenta el número de víctimas. Por esto, es de mucha utilidad establecer el tiempo que se tardó en duplicarse el número to- 
Tabla 2. Definición de algunos conceptos que deben tenerse en cuenta al analizar los modelos epidemiológicos de una pandemia

\begin{tabular}{|c|c|}
\hline $\begin{array}{l}\text { Vigilancia } \\
\text { epidemiológica }\end{array}$ & $\begin{array}{l}\text { Es el análisis, interpretación y difusión sistemática de los datos recolectados, utilizando métodos que se } \\
\text { distinguen por ser prácticos, uniformes y rápidos, que sirven para observar las tendencias en tiempo, lugar } \\
\text { y persona, con lo que pueden anticiparse cambios para realizar las acciones oportunas, incluyendo la in- } \\
\text { vestigación y/o la aplicación de medidas de control o de intervención. }\end{array}$ \\
\hline Curva epidémica & $\begin{array}{l}\text { Es la representación gráfica del número de casos de una enfermedad según la fecha de inicio de los sínto- } \\
\text { mas. La curva epidémica tiene, usualmente, una distribución asimétrica y presenta los siguientes elemen- } \\
\text { tos: la curva ascendente, que representa la fase de crecimiento de la epidemia y cuya pendiente o grado de } \\
\text { inclinación indica la velocidad de propagación de la epidemia, que está asociada al modo de transmisión } \\
\text { del agente y al tamaño de la población susceptible. El punto máximo o meseta, que puede ser alcanzado } \\
\text { naturalmente o truncado por una intervención temprana. La curva descendente representa la fase de ago- } \\
\text { tamiento de la epidemia y cuya pendiente o grado de inclinación indica la velocidad de agotamiento de } \\
\text { la población susceptible, sea naturalmente o por efecto o impacto de las medidas de control establecidas. }\end{array}$ \\
\hline $\begin{array}{l}\text { Transmisión por } \\
\text { contacto estrecho }\end{array}$ & $\begin{array}{l}\text { Se trata de aquellos casos confirmados que han estado expuestos durante el período de incubación a la } \\
\text { transmisión proveniente de una persona con diagnóstico confirmado de una enfermedad. Por su parte, un } \\
\text { contacto estrecho es cualquier persona que haya permanecido a una distancia menor de un metro con una } \\
\text { persona que presentaba síntomas y luego fue confirmada por coronavirus. }\end{array}$ \\
\hline $\begin{array}{l}\text { Transmisión } \\
\text { comunitaria }\end{array}$ & $\begin{array}{l}\text { Se trata de casos en los cuales no puede establecerse ningún nexo epidemiológico con algún caso confir- } \\
\text { mado o con un conglomerado de casos y que, por lo tanto, se supone que la infección fue adquirida en el } \\
\text { ámbito de la comunidad. }\end{array}$ \\
\hline $\begin{array}{l}\text { Número } \\
\text { reproductivo } \\
\text { básico }(\mathrm{R} 0)\end{array}$ & $\begin{array}{l}\text { Mide la transmisibilidad intrínseca de un agente infeccioso al comienzo de una epidemia cuando toda la po- } \\
\text { blación es susceptible. El R0 indica el número de infecciones secundarias producidas por un caso, durante su } \\
\text { período de infectividad, en una población completamente susceptible. El número dependerá del número de } \\
\text { contactos entre las personas por unidad de tiempo, de la duración de la infectividad y de la probabilidad de } \\
\text { que ocurra la transmisión tras contactar con una persona infectada en el período de infectabilidad. }\end{array}$ \\
\hline $\begin{array}{l}\text { Tasa de ataque del } \\
\text { patógeno }\end{array}$ & Es la proporción de individuos de una población específica, los cuales se enfermarían durante un brote. \\
\hline $\begin{array}{l}\text { Período de latencia } \\
\text { y de incubación }\end{array}$ & $\begin{array}{l}\text { En las enfermedades transmisibles, el período de latencia es el tiempo que transcurre desde la infección } \\
\text { hasta que el individuo se vuelve infeccioso. El período de incubación es el tiempo que transcurre desde la } \\
\text { infección hasta la presentación de síntomas. }\end{array}$ \\
\hline $\begin{array}{l}\text { Período de } \\
\text { transmisibilidad o } \\
\text { infeccioso }\end{array}$ & $\begin{array}{l}\text { Es el intervalo de tiempo durante el cual el agente infeccioso puede ser transferido directa o indirectamen- } \\
\text { te de un individuo infectado a otro, o de un animal infectado a un humano, o de un humano infectado a } \\
\text { un animal. }\end{array}$ \\
\hline $\begin{array}{l}\text { Tasa o índice de } \\
\text { letalidad }\end{array}$ & Es el cociente de desenlaces fatales en relación con las personas que se han contagiado por la enfermedad. \\
\hline Aislamiento social & $\begin{array}{l}\text { Se refiere al enfermo; es la separación de personas con una enfermedad infecciosa específica, en sitios } \\
\text { como el hogar, los hospitales, entre otros. }\end{array}$ \\
\hline $\begin{array}{l}\text { Distanciamiento } \\
\text { social }\end{array}$ & $\begin{array}{l}\text { Son las medidas de prevención diseñadas para evitar la propagación del SARS-CoV-2, como el cambio de } \\
\text { rutinas laborales, culturales, sociales, etc., para reducir el riesgo de contagio. Estos esfuerzos se traducen en } \\
\text { aspectos como: trabajar desde la casa, no usar transporte público, alejarse de otras personas en el espacio } \\
\text { público, evitar reuniones presenciales, aglomeraciones, entre otros. }\end{array}$ \\
\hline Cuarentena & $\begin{array}{l}\text { Se refiere a los contactos del enfermo; es aplicable en algunas enfermedades que se transmiten de persona } \\
\text { a persona. Este término se aplica para las personas que no tienen síntomas, pero que probablemente han } \\
\text { estado en contacto con el virus. }\end{array}$ \\
\hline $\begin{array}{l}\text { Estrategia de } \\
\text { contención }\end{array}$ & $\begin{array}{l}\text { Contener el virus significa cerrarle el paso para evitar que se propague. Usualmente esto se hace al prin- } \\
\text { cipio de la epidemia y consiste en identificar y aislar los casos confirmados, así como rastrear y poner en } \\
\text { cuarentena a todas las personas con las que pueden haber interactuado. Para que una contención sea } \\
\text { exitosa, debe hacerse un testeo adecuado, con un número diario alto de pruebas. }\end{array}$ \\
\hline $\begin{array}{l}\text { Estrategia de } \\
\text { "mitigación" }\end{array}$ & $\begin{array}{l}\text { Se enfoca en desacelerar, sin poder detener la propagación del virus, reduciendo la demanda de atención } \\
\text { médica y protegiendo a los individuos con mayor riesgo de enfermedad grave. }\end{array}$ \\
\hline $\begin{array}{l}\text { Estrategia de } \\
\text { "supresión" }\end{array}$ & $\begin{array}{l}\text { Se enfoca en revertir el crecimiento epidémico, reduciendo el número de casos a niveles bajos, mantenien- } \\
\text { do esa situación indefinidamente, mediante un paquete de medidas de aislamiento total y testeo de casos } \\
\text { en forma masiva. }\end{array}$ \\
\hline
\end{tabular}


tal de muertes confirmadas; actualmente, el promedio mundial de la velocidad a la cual se duplica el número de muertes confirmadas por COVID-19 es de 41 días (a nivel mundial), y para Colombia se ha establecido, hasta ahora, en 21 días ${ }^{(27) .}$

\section{Testeo poblacional para la COVID-19}

Hasta la fecha, ningún país conoce el número total de personas infectadas con COVID-19, puesto que es virtualmente imposible testear al total de la población, por lo que solo conocemos el estado de infección de aquellos individuos que han sido testeados. Todos los que tienen una infección confirmada por el laboratorio se cuentan como casos confirmados. Esto significa que los recuentos de casos confirmados dependen de cuántas pruebas se realizan en un país. Sin un protocolo universal para realizar pruebas, no habría datos poblacionales, evidentemente. El testeo es nuestro pasaporte hacia la pandemia, con el fin de determinar la forma de propagación. Sin disponer de datos sobre quién está infectado por el virus, no tenemos forma de entender la pandemia. Para poder interpretar cualquier dato sobre los casos confirmados, es necesario saber cuántas pruebas de COVID-19 hace realmente el país. Los datos para el 2 de junio, en Colombia, indican que se realizan 0,22 pruebas diarias por cada 1000 habitantes, a diferencia de países como Chile, en donde, para la misma fecha, se realizan diariamente 0,83 pruebas por 1000 habitantes; sin embargo, se supera a países como Paraguay, Perú, Argentina, Ecuador y Brasil. No obstante, el número de pruebas no significa tácitamente lo mismo en cada país; por ejemplo, algunos países informan la cantidad de personas testeadas, mientras que otros informan la cantidad de pruebas realizadas (que puede ser mayor si un individuo se testea más de una vez), y otros países informan sobre dichas pruebas de una manera que no deja claro a qué se refieren exactamente ${ }^{(28)}$. El número total de personas con pruebas reportadas como positivas (es decir, el número de casos confirmados) no es el número total de personas infectadas. Adicionalmente, se debe tener en cuenta que, al comienzo de un brote, cuando el número de personas infectadas con el virus es bajo, se necesita un número mucho menor de pruebas para evaluar con precisión la propagación del virus, pero a medida que el proceso se expande en la población, también es necesario ampliar la cobertura de las pruebas; por ello, también es útil evaluar el número de pruebas realizadas para cada caso confirmado. Éste es un indicador de la escala de las pruebas que explican las diferentes etapas del brote en la que un área geográfica específica se encuentra. En algunos países, el número de pruebas es muchas veces mayor que el número de casos confirmados, y en otros, el número de pruebas es muy bajo en relación con el número de casos confirmados. Hasta el 2 de junio, en Nueva Zelanda se habían realizado casi 247,5 pruebas por cada caso confirmado, en Australia se habían realizado 206,9 pruebas por cada caso confirmado, y en Colombia, 11,5 pruebas. Tanto la cobertura de pruebas como la can- tidad de pruebas por caso confirmado permite comprender la propagación del virus a partir de los casos confirmados, pero, probablemente, el número de pruebas por caso confirmado sea el dato más útil, y podría explicar también el hecho del por qué un brote más pequeño requiere menos pruebas. Así, un país que realiza muy pocas pruebas para cada caso confirmado realmente no está llevando a cabo la suficiente cantidad de pruebas como para que el número de casos confirmados arroje una imagen confiable de la verdadera propagación del virus. Si bien las personas con los síntomas más graves pueden haber sido analizadas en dichos países, es probable que haya muchas más personas con síntomas leves o sin síntomas que nunca fueron analizados. Por lo tanto, cuando el número de casos confirmados parece bajo en comparación con el número de muertes, esto es un claro indicio de que es probable que el número real de casos sea mucho mayor (aunque la causa fundamental para ello sea el número limitado de pruebas realizadas) ${ }^{(16,29)}$.

\section{Tasa de letalidad y mortalidad por COVID-19}

La tasa de letalidad también se conoce como riesgo de letalidad o razón de letalidad, y se calcula dividiendo el total de muertes confirmadas por la enfermedad (en este caso, por COVID-19) sobre el número total de casos confirmados; por ejemplo, si el total de muertos por COVID-19 es de 25 y el total de individuos confirmados con la enfermedad fue de 250 , entonces, la tasa de letalidad es: 25/250; es decir, 0,1 (10\%). La tasa de letalidad no es un parámetro constante, sino que refleja la gravedad de la enfermedad en un contexto, momento y población particular, ya que la probabilidad de que un individuo muera por una enfermedad no solo depende de la enfermedad en sí, sino de aspectos como el tratamiento que recibe y de la capacidad que tiene para recuperarse dicha enfermedad. Por lo anterior, la tasa de letalidad puede disminuir o aumentar con el tiempo, a medida que cambian las intervenciones y las respuestas a ellas, y puede variar según la ubicación y las características de la población infectada (como la edad, el sexo, las comorbilidades de base, entre otras) ${ }^{(30)}$. Así, las poblaciones mayores de 70 años tienen una mayor tasa de letalidad que las menores de dicha edad (Figura 2). La tasa de letalidad a nivel mundial por COVID-19 es del 6\% y en Colombia, del 3,17\% (junio 3 de 2020). Por su parte, la tasa cruda de mortalidad nos dice la probabilidad de que cualquier individuo dentro de una población muera por una enfermedad, y no solo en aquellos que están infectados, o en los que se confirma que están infectados (por ende, se toma como denominador al total de la población). Por ejemplo, si hubo 30 muertes en una población de 1000 individuos, la tasa cruda de mortalidad sería de 30/1000; es decir 0,03 (3\%). Lo anterior indica que la tasa de letalidad será siempre mayor que la tasa cruda de mortalidad. Por otro lado, la tasa de mortalidad por la infección se refiere al número de muertes por una enfermedad di- 
vidido por el número total de casos. Por lo que, si 20 personas mueren por COVID-19 y 500 personas la padecen, la tasa de mortalidad por la infección (COVID-19) será de 20/500 o 0,04 (4\%). El problema es que en la vida real no se conoce con precisión el número total de casos con la enfermedad, ya que, por ejemplo, la mayor parte de los asintomáticos o sintomáticos leves no es testeada y hasta ahora se tiene claro que el virus se propaga muy rápido una vez que se introduce dentro de una población. Esto significa que un número importante de personas en la población general podría estar o ya ha sido infectada con el virus (con síntomas o sin síntomas). Sin embargo, en lugar de contar a todas las personas infectadas en el denominador, en muchos países, incluido Colombia, solo se tiene en cuenta a las personas lo suficientemente enfermas como para ir a un hospital (o a buscar asistencia médica). Esto, a la larga, es un sesgo de selección, ya que las personas que están lo suficientemente enfermas como para buscar asistencia médica tienen más probabilidades de requerir atención (en unidades de cuidados intensivos, por ejemplo) que los pacientes con síntomas leves. Adicionalmente, incluso a pesar de que se están realizando pruebas (dependiendo del tipo de pruebas utilizadas y de cómo las estamos utilizando), es posible que solo se esté contando a las personas infectadas activamente. Esto también conduce a una subestimación del denominador, lo cual significa que el denominador (número de infecciones) es más pequeño de lo que realmente es, por lo que el numerador (número de muertes) asume un gran poder en el resultado de la ecuación. En este caso, la tasa de mortalidad por infección (numerador dividido por denominador) es más alta de lo que debería ser. En otras palabras: al contar solo a las personas que acuden a los servicios de salud, se está sobreestimando la proporción de personas infectadas que mueren por COVID-19. En las próximas semanas, en Colombia, la tasa de mortalidad se describirá mucho más alta que la actual, especialmente en la medida que los hospitales se llenen cada vez más y tengan que racionalizar la atención a los pacientes ${ }^{(31-33)}$.

\section{Número reproductivo básico (R0)}

El R0 es un parámetro teórico, el cual proporciona cierta información sobre la velocidad con que una enfermedad puede propagarse en una población determinada, de modo que un valor umbral de 1,0 indica cuándo ocurrirá un brote. Si R0 $>1,0$, entonces, es una epidemia; si R0 =1,0, es endemia; y si es $<1,0$, existiría control de la enfermedad. El R0 posee tres componentes, a saber: (b): la tasa de ataque (para COVID-19 se estima entre 60\%-80\%); (c): número de contactos potencialmente infecciosos de un caso (no hay datos en nuestro medio, pero extrapolando datos de población europea, podría ser de 30 contactos/semanales); (d): duración de la transmisibilidad, que actualmente se considera que para infectados asintomáticos es de 14 días (2 semanas); para sintomáticos leves, de 21 días (3 semanas); y para sintomáticos graves o críticos, de 28 a 36 días (4 semanas). La fórmula para R0 es: $\mathrm{R} 0=\mathrm{b} \times \mathrm{c} \times \mathrm{d}$. El R0 a nivel mundial es muy variable, este puede estar entre 1,5 y 3,5 . Sin embargo, este valor es cambiante desde que comienza la epidemia y disminuye con la aplicación de medidas de salud pública (distanciamiento social, cuarentena, entre otras) ${ }^{(35,36)}$.

\section{Proyecciones y estrategias de salud pública en el abordaje de la pandemia por COVID-19 en Colombia}

Las estrategias de salud pública se establecen con el propósito de reducir el número de individuos infectados $y$, por lo tanto, el número de personas que requerirían eventualmente manejo intrahospitalario. Para ello, los modelos matemáticos que proyectan y predicen la dinámica de la pandemia en la población aportan una gran información al respecto. Un modelo matemático estándar parte del supuesto de que los individuos se encuentran en una de varias categorías posibles: individuos susceptibles (S), expuestos (E), infectados (I), recuperados $(\mathrm{R})$, entre otros. En estos modelos, se asume que la interacción entre los individuos es aleatoria. En un modelo SIR, por

Figura 2. Tasas de letalidad entre todos los infectados según la edad (sintomáticos/asintomáticos). Fuente: adaptada de la referencia 34 .

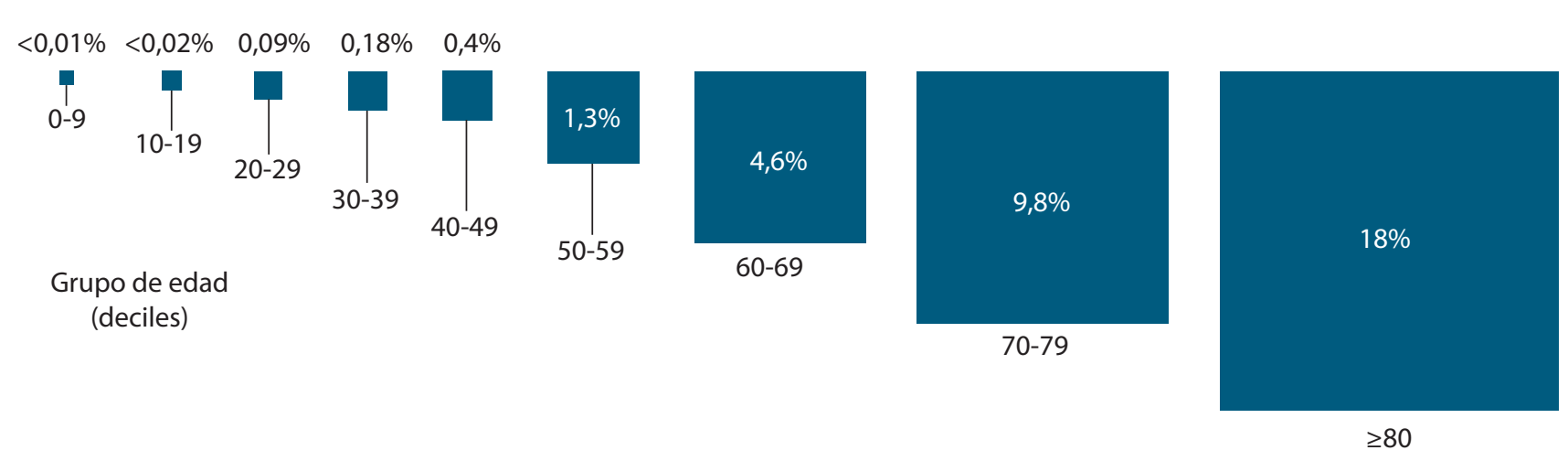


ejemplo, los individuos pueden pasar de ser susceptibles a infecciosos y, de allí, a resistentes. Asimismo, en un modelo SIS, los individuos pueden pasar de ser susceptibles a ser infecciosos y, de allí, a ser susceptibles de nuevo. Los modelos que deben utilizarse dependerán del patógeno y de la forma como se transmite, ya que estos varían de una enfermedad a otra. Para Colombia, se han diseñado varios de estos modelos que plantean varios escenarios posibles. Cada uno de ellos parte de supuestos como "el peor escenario posible" en el cual la población no realiza ninguna estrategia poblacional de control (aislamiento social, uso de tapabocas, entre otros) o "el mejor escenario posible" en donde se asume que gran parte de la población adopta medidas de aislamiento, lavado de manos, uso de tapabocas, entre otros ${ }^{(37)}$. Los parámetros que se utilizan en los modelos dependen de los datos disponibles, por lo que pueden irse modificando según la evolución de la pandemia. Dichos parámetros derivan de las actividades de vigilancia epidemiológica o de los estudios de campo, al igual que de la experiencia previa en relación con el efecto de un patógeno sobre la población; por eso, si el modelo se lleva a cabo al inicio de una pandemia, puede haber datos no disponibles, por ejemplo, el R0, la velocidad con la que se duplican los casos, la tasa de ataque, entre otros; en ese momento, la incertidumbre de los modelos es más alta y, en general, se tiende a establecer supuestos con "el peor escenario posible" $(38,39)$.
A partir de estos modelos se pueden establecer recomendaciones poblacionales desde los diferentes entes gubernamentales y de la salud pública, las cuales intentan reducir el número de casos y la velocidad con que estos se presentan. La idea de estas recomendaciones y/o estrategias es "aplanar" la curva epidémica, con el propósito de que el número total de casos no se presente en un corto período, sino en un período más "larvado", permitiéndole al sistema de salud tener una mejor capacidad de respuesta para la atención de los pacientes enfermos (Figura 3) ${ }^{(40,41)}$.

En cuanto a las estrategias recomendadas desde el punto de vista de la salud pública, se destacan, entre otras, el aislamiento social, la cuarentena, la contención, la mitigación y la supresión; todas ellas son medidas no farmacológicas ${ }^{(41)}$. A partir del 30 de enero de 2020, la OMS determinó que la COVID-19 constituía una emergencia de salud pública de importancia internacional. Los países de la región de las Américas implementaron inicialmente medidas destinadas a restringir la entrada de viajeros internacionales procedentes de países específicos, en los cuales se observaba la transmisión de COVID-19. Coincidiendo con la propagación de la COVID-19 a Europa a finales de febrero de 2020 y, posteriormente, con la declaración de la pandemia asociada con COVID-19 por la OMS el 11 de marzo de 2020, estas medidas se volvieron progresivamente más restrictivas. Las medidas de distanciamiento social se aplican a individuos (por

Figura 3. Aplanamiento de la curva. Se muestra el efecto de las medidas de intervención sobre la ralentización del número de casos y su efecto sobre el sistema de salud (véase el texto para más detalles). Fuente: adaptada de la referencia 41.

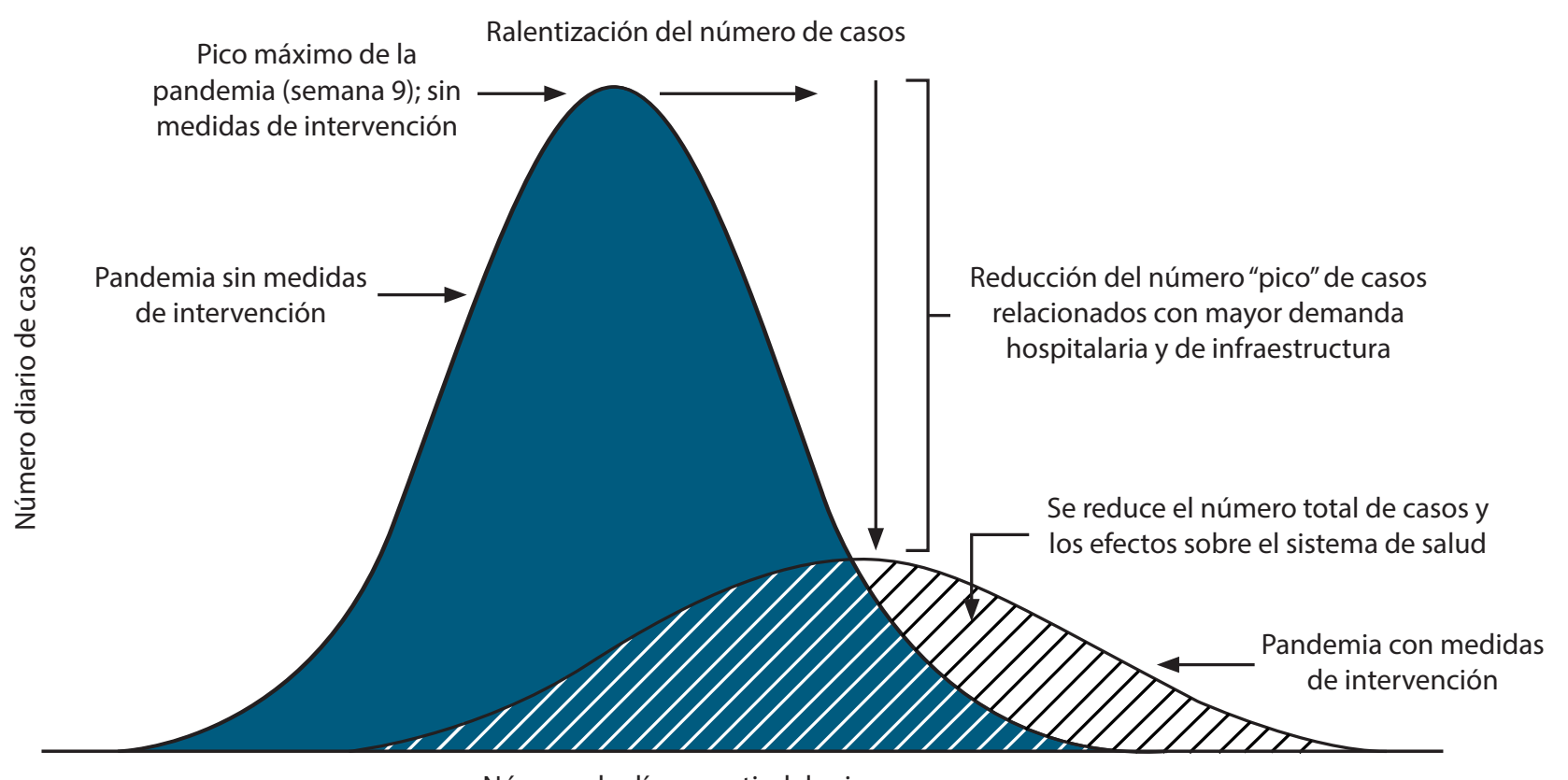

Número de días a partir del primer caso 
ejemplo, aislamiento de casos y cuarentena de contactos) o a la comunidad (a segmentos específicos de la población, por ejemplo, confinamiento en un hogar para ancianos; o a la población en general, por ejemplo, confinamiento en el hogar y cierre de todos los negocios no esenciales). Estas medidas no son mutuamente excluyentes ${ }^{(42,43)}$.

\section{Utilidad de las medidas de distanciamiento social}

El distanciamiento social tiene como objetivo, y a través de una variedad de medios, minimizar el contacto físico entre las personas y, por lo tanto, reducir la posibilidad de nuevas infecciones. Las decisiones sobre cuándo y cómo implementar medidas de distanciamiento social (y a nivel comunitario, en paralelo) siempre deben basarse en evidencia (pero rara vez se basarán únicamente en evidencia). También deberán tenerse en cuenta las consideraciones sociales y políticas. La detección de casos o muertes por COVID-19 fuera de las cadenas de transmisión conocidas es una fuerte señal de que medidas como el distanciamiento social, el cierre de lugares de trabajo/escuelas, la cancelación de reuniones masivas y la cuarentena de las áreas afectadas deberían considerarse ${ }^{(44)}$. La implementación temprana, decisiva, rápida, coordinada e integral de medidas, cierres y cuarentenas es probable que sea más efectiva para frenar la propagación del virus que una implementación tardía. Se deben realizar esfuerzos de contención (por ejemplo, rastreando contactos), cada vez que la contención por sí sola ya no sea suficiente como un medio para retrasar el pico de la epidemia; es necesario, además, disminuir la magnitud de la pandemia (el pico) para proteger la capacidad de atención médica o proteger a los grupos vulnerables en riesgo de sufrir desenlaces graves o fatales. Existen varios tipos diferentes de medidas de distanciamiento social (Tabla 2) que se pueden clasificar en "capas" estipuladas, usualmente en orden ascendente ${ }^{(45)}$. Es importante tener en cuenta que el término distanciamiento social se centra en reducir el contacto físico como un medio de interrumpir la transmisión, pero si bien la reducción del contacto social puede ser el resultado de eso, no es un objetivo específico. De hecho, el éxito de las medidas de distanciamiento social que se implementan durante un período prolongado puede depender de garantizar a las personas que mantengan un contacto social desde la distancia, con amigos, familiares, compañeros, colegas, entre otros (46). No existe un método único para decidir el momento preciso para establecer las medidas de distanciamiento social que impliquen cierres de escuelas, universidades o la cuarentena de áreas afectadas. En una situación epidemiológica en donde se documente un brote localizado que comienza a fusionarse, o cuando hay una transmisión sostenida y generalizada, o cuando la detección de casos de la enfermedad o de muertes fuera de una cadena conocida de transmisión están presentes, proporciona una señal de que las medidas de distanciamiento social deberían implementarse. Además, los datos sobre el número de casos apoyan la implementación simultánea de varias "capas o etapas" de distanciamiento social a la vez (en lugar de una por una). Mientras tanto, como un medio para facilitar la aceptación pública de las medidas de distanciamiento social, es importante que se establezca y comunique una fecha de finalización anticipada lo antes posible. También debe quedar claro para la población que las medidas podrían ampliarse si las circunstancias así lo requieren, y que algunas medidas pueden eliminarse o reducirse mientras que otras permanecen constantes. Además, se deben elaborar planes y comunicar al público en general que existe la posibilidad de volver a imponer medidas de distanciamiento a gran escala si hay un resurgimiento de la transmisión después del levantamiento de las medidas $(47,48)$.

\section{Escenarios futuros de la pandemia}

Al proyectar los posibles escenarios futuros de la pandemia, estos han sido planteados en un concepto denominado olas y pueden resumirse de la siguiente forma (Figura 4):

- Escenario 1 (picos y valles): la primera ola (en la primavera de 2020) es seguida por una serie de olas repetitivas más pequeñas durante el verano, y luego consistentemente durante un período de 1 a 2 años, disminuyendo gradualmente en algún momento en el 2021. La ocurrencia de estas olas puede variar geográficamente y puede depender de las medidas de mitigación que se estén implementando. Dependiendo de qué tan altos sean los picos de las olas, este escenario podría requerir la reinstitución periódica de las medidas de mitigación y de la posterior relajación de estas en los próximos 1 a 2 años.

- Escenario 2 (pico de otoño): la primera ola (en la primavera de 2020) es seguida por una ola más grande en el otoño o el invierno de 2020 y una o más olas posteriores (más pequeñas) en el 2021. Este patrón puede requerir la reinstitución de las medidas de mitigación en el otoño, en un intento por reducir la propagación de la infección y evitar que los sistemas de salud colapsen.

- Escenario 3 (pico y lenta combustión): la primera ola (en la primavera de 2020) es seguida por una "lenta combustión" de la transmisión de la enfermedad y de la ocurrencia de casos, pero sin un patrón claro de las ondas. Este patrón puede variar geográficamente y puede estar influenciado por las medidas de mitigación implementadas. Este tercer escenario probablemente no requeriría la reinstitución de las medidas de mitigación, aunque los casos y las muertes continuarían ocurriendo ${ }^{(49,50)}$. 
Figura 4. Posibles escenarios futuros y olas pandémicas por COVID-19. Fuente: adaptada de la referencia 49.
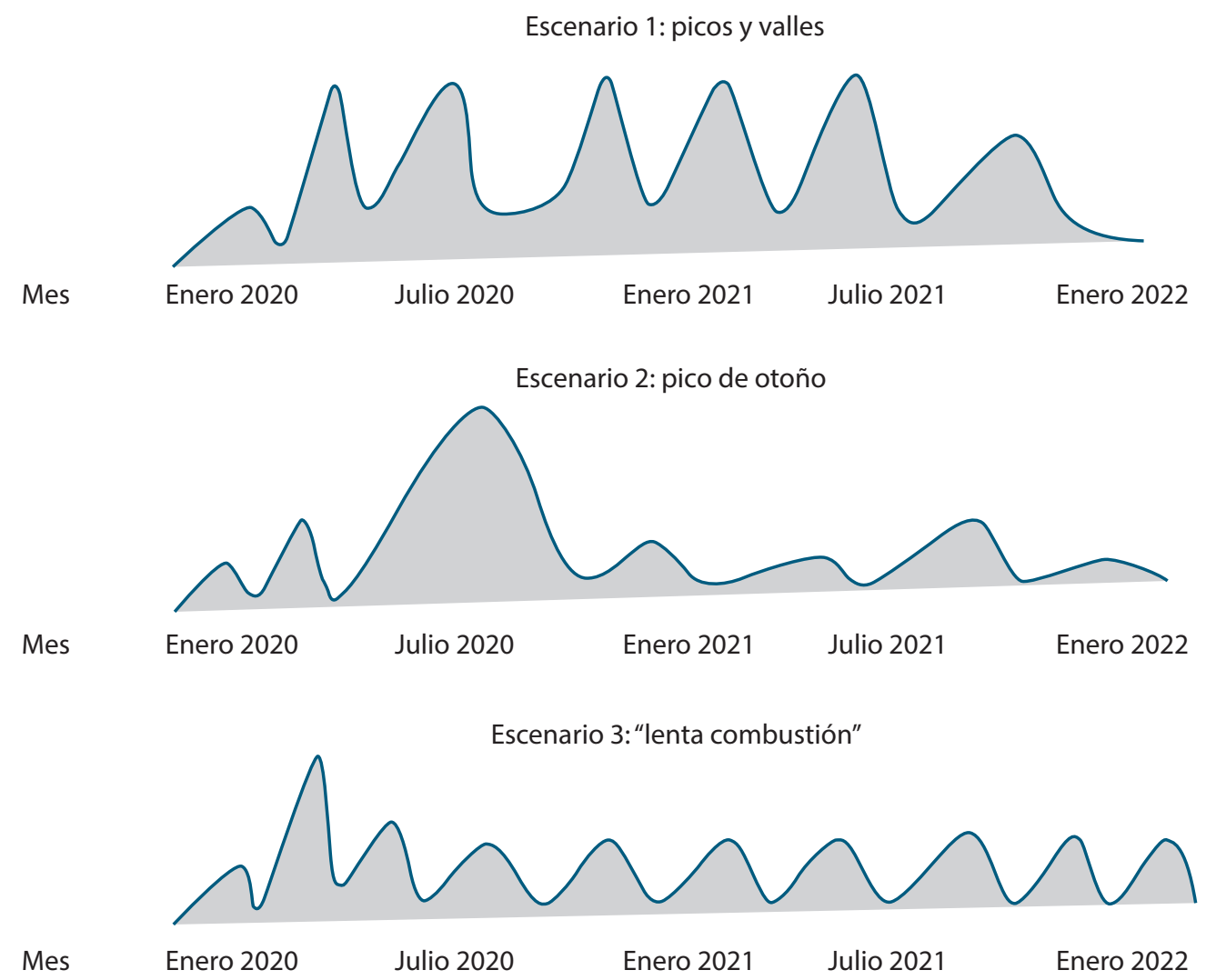

Cualquiera sea el escenario que siga la pandemia (suponiendo al menos algún nivel de medidas de mitigación en curso), debemos estar preparados (al menos) por otros 18 a 24 meses de actividad de la COVID-19, con apariciones o brotes periódicos en diversas áreas geográficas. A medida que la pandemia vaya disminuyendo, es probable que el SARS-CoV-2 continúe circulando dentro de la población y probablemente se sincronizaría con un patrón "estacional" y con una severidad disminuida a través del tiempo (al igual que con otros coronavirus menos patógenos y con el virus de la influenza) ${ }^{(51)}$.

\section{Conclusiones}

La pandemia por SARS-CoV-2 ha sido una prueba de fuego para los diferentes sistemas de salud. El esfuerzo mancomunado entre la realización masiva de pruebas diagnósticas, los modelos de proyección, las decisiones basadas en la evidencia y en la experiencia probablemente sean la mejor estrategia para el abordaje de esta enfermedad. Los estados, los territorios y las autoridades de salud deben planificar desde el "mejor" hasta el "peor" de los casos, incluido el hecho de no disponer de una vacuna con efectividad comprobada. Los entes gubernamentales, con poder de decisión, deben desarrollar planes concretos que incluyan factores determinantes para establecer las medidas de mitigación (para tratar los picos de la enfermedad cuando ocurran). Finalmente, la forma de comunicar las diferentes estrategias y medidas poblacionales debe incorporar el concepto de que esta pandemia no terminará pronto y que las personas deben estar preparadas para posibles resurgimientos periódicos de la enfermedad en los próximos 2 años.

\section{Conflicto de interés}

Los autores declaran no tener conflictos de intereses en el desarrollo del documento.

\section{Financiación}

Los autores declaran no haber recibido financiación de alguna índole para el desarrollo de este documento. 


\section{Referencias}

1. Wu F, Zhao S, Yu B, Chen YM, Wang W, Song ZG, et al. A new coronavirus associated with human respiratory disease in China. Nature. 2020;579(7798):265-9. doi: 10.1038/s41586-020-2008-3.

2. Wu F, Zhao S, Yu B, Chen YM, Wang W, Song ZG, et al. Wuhan seafood market pneumonia virus isolate Wuhan-Hu-1, complete genome. 23 de enero de 2020 [citado 09 de mayo de 2020]; Disponible en: http://www.ncbi. nlm.nih.gov/nuccore/MN908947.3.

3. World Health Organization. Disease outbreaks by year [Internet]. Citado abril 19 de 2020. Disponibles en: https://www.who.int/csr/don/archive/ year/en/.

4. Graham RL, Baric RS. SARS-CoV-2: Combating Coronavirus Emergence. Immunity. 2020;52(5):734-6. doi: 10.1016/j.immuni.2020.04.016.

5. Wu J, Huang Y, Tu C, Bi C, Chen Z, Luo L, et al. Household Transmission of SARS-CoV-2, Zhuhai, China, 2020. Clin Infect Dis. 2020;ciaa557. doi: 10.1093/cid/ciaa557.

6. Li Y, Wang J, Wang C, Yang Q, Xu Y, Xu J, et al. Characteristics of respiratory virus infection during the outbreak of 2019 novel coronavirus in Beijing. Int J Infect Dis. 2020;96:266-9. doi: 10.1016/j.ijid.2020.05.008.

7. Liu YC, Kuo RL, Shih SR. COVID-19: The First Documented Coronavirus Pandemic in History. Biomed J. 2020;S2319-4170(20)30044-5. doi: 10.1016/j.bj.2020.04.007.

8. Chang CL, McAleer M. Alternative Global Health Security Indexes for Risk Analysis of COVID-19. Int J Environ Res Public Health. 2020;17(9)E3161. doi: $10.3390 /$ ijerph17093161.

9. World Health Organization. Coronavirus [Internet]. [Citado 01 de mayo de 2020]. Disponible en: https://www.who.int/health-topics/coronavirus.

10. World Health Organization. Coronavirus disease (COVID-2019) situation reports [Internet]. [Citado 10 de mayo de 2020]. Disponible en: https:// www.who.int/emergencies/diseases/novel-coronavirus-2019/situationreports.

11. Paules CI, Marston HD, Fauci AS. Coronavirus Infections -- More Than Just the Common Cold. JAMA. 2020. doi: 10.1001/jama.2020.0757.

12. Cyranoski D. Mystery deepens over animal source of coronavirus. Nature. 2020;579(7797):18-9. doi: 10.1038/d41586-020-00548-w.

13. Lai CC, Wang CY, Wang YH, Hsueh SC, Ko WC, Hsueh PR. Global epidemiology of coronavirus disease 2019 (COVID-19): disease incidence, daily cumulative index, mortality, and their association with country healthcare resources and economic status. Int J Antimicrob Agents. 2020;55(4):105946. doi: 10.1016/j.ijantimicag.2020.105946.

14. Wang K, Zuo P, Liu Y, Zhang M, Zhao X, Xie S, et al. Clinical and laboratory predictors of in-hospital mortality in patients with COVID-19: a cohort study in Wuhan, China. Clin Infect Dis. 2020;ciaa538. doi: 10.1093/cid/ ciaa538.

15. Yu X, Sun X, Cui P, Pan H, Lin S, Han R, et al. Epidemiological and Clinical Characteristics of 333 Confirmed Cases with Coronavirus Disease 2019 in Shanghai, China. Transbound Emerg Dis. 2020. doi: 10.1111/tbed.13604.

16. Back D, Marzolini C, Hodge C, Marra F, Boyle A, Gibbons S, et al. COVID-19 treatment in patients with comorbidities: Awareness of drug-drug interactions. Br J Clin Pharmacol. 2020. doi: 10.1111/bcp.14358.

17. Roser M, Ritchie H, Ortiz-Ospina E, Hasell J. Coronavirus Pandemic (COVID-19). [citado 3 de junio de 2020]. Disponible en: https://ourworldindata.org/coronavirus.

18. LePan N. Visualizing the History of Pandemics [Internet]. [Citado 07 de mayo de 2020]. Disponible en: https://www.visualcapitalist.com/historyof-pandemics-deadliest/.

19. Erduran S. Science Education in the Era of a Pandemic: How Can History, Philosophy and Sociology of Science Contribute to Education for Understanding and Solving the Covid-19 Crisis? Sci Educ (Dordr). 2020:1-3. doi: 10.1007/s11191-020-00122-w.

20. Hunter P. The spread of the COVID-19 coronavirus: Health agencies worldwide prepare for the seemingly inevitability of the COVID-19 coronavirus becoming endemic. EMBO Rep. 2020;21(4):e50334. doi: 10.15252/ embr.202050334

21. Graham BS, Corbett KS. Prototype pathogen approach for pandemic preparedness: world on fire. J Clin Invest. 2020;139601. doi: 10.1172/ JCI139601.

22. Yuen KS, Ye ZW, Fung SY, Chan CP, Jin DY. SARS-CoV-2 and COVID-19: The most important research questions. Cell Biosci. 2020;10:40. doi: 10.1186/ s13578-020-00404-4.

23. Li H, Liu SM, Yu XH, Tang SL, Tang CK. Coronavirus disease 2019 (COVID-19): Current status and future perspectives. Int I Antimicrob Agents. 2020;55(5):105951. doi: 10.1016/j.ijantimicag.2020.105951.

24. Najafimehr H, Mohamed Ali K, Safari S, Yousefifard M, Hosseini M. Estimation of basic reproduction number for COVID-19 and the reasons for its differences. Int J Clin Pract. 2020. doi: 10.1111/ijcp.13518.

25. Akin L, Gözel MG. Understanding dynamics of pandemics. Turk J Med Sci. 2020;50(SI-1):515-9. doi: 10.3906/sag-2004-133.
26. Rosenberg ES, Dufort EM, Blog DS, Hall EW, Hoefer D, Backenson BP et al. COVID-19 Testing, Epidemic Features, Hospital Outcomes, and Household Prevalence, New York State-March 2020. Clin Infect Dis. 2020;ciaa549. doi: $10.1093 / \mathrm{cid} / \mathrm{ciaa} 549$.

27. Ahn DG, Shin HJ, Kim MH, Lee S, Kim HS, Myoung J, et al. Current Status of Epidemiology, Diagnosis, Therapeutics, and Vaccines for Novel Coronavirus Disease 2019 (COVID-19). J Microbiol Biotechnol. 2020;30(3):313-24. doi: 10.4014/jmb.2003.03011.

28. Yin S, Peng Y, Ren Y, Hu M, Tang L, Xiang Z, et al. The implications of preliminary screening and diagnosis: Clinical characteristics of 33 mild patients with SARS-CoV-2 infection in Hunan, China. J Clin Virol. 2020;128:104397. doi: 10.1016/j.jcv.2020.104397.

29. Eberhardt JN, Breuckmann NP, Eberhardt CS. Multi-Stage Group Testing Improves Efficiency of Large-Scale COVID-19 Screening. J Clin Virol 2020;128:104382. doi: 10.1016/j.jcv.2020.104382.

30. Mi YN, Huang TT, Zhang JX, Qin Q Gong YX, Liu SY, et al. Estimating in stant case fatality rate of COVID-19 in China. Int J Infect Dis. 2020;S1201 9712(20)30271-X. doi: 10.1016/j.ijid.2020.04.055.

31. Verity R, Okell LC, Dorigatti I, Winskill P, Whittaker C, Imai N, et al. Estimates of the severity of coronavirus disease 2019: a model-based analysis. Lancet Infect Dis. 2020;S1473-3099(20)30243-7. doi: 10.1016/S14733099(20)30243-7.

32. Manski CF, Molinari F. Estimating the COVID-19 infection rate: Anatomy of an inference problem. J Econom. 2020. doi: 10.1016/j.jeconom.2020.04.041.

33. Medeiros de Figueiredo A, Daponte A, Moreira Marculino de Figueiredo DC, Gil-García E, Kalache A. [Case fatality rate of COVID-19: absence of epidemiological pattern]. Gac Sanit. 2020;S0213-9111(20)30084-4. doi: 10.1016/j.gaceta.2020.04.001.

34. Riou J, Hauser A, Counotte MJ, Althaus CL. Adjusted age-specific case fatality ratio during the COVID-19 epidemic in Hubei, China, January and February 2020. medRxiv. 2020. doi: https://doi.org/10.1101/2020.03.0 4.20031104.

35. Viceconte G, Petrosillo N. COVID-19 R0: Magic number or conundrum? In fect Dis Rep. 2020;12(1):8516. doi: 10.4081/idr.2020.8516.

36. Hens N, Vranck P, Molenberghs G. The COVID-19 epidemic, its mortality, and the role of non-pharmaceutical interventions. Eur Heart J Acute Cardiovasc Care. 2020;2048872620924922. doi: 10.1177/2048872620924922.

37. Kucharski AJ, Russell TW, Diamond C, Liu Y, Edmunds J, Funk S, et al. Centre for Mathematical Modelling of Infectious Diseases COVID-19 working group. Early dynamics of transmission and control of COVID-19: a mathematical modelling study. Lancet Infect Dis. 2020;20(5):553-8. doi: 10.1016/S1473-3099(20)30144-4

38. Yan Y, Shin WI, Pang YX, Meng Y, Lai J, You C, et al. The First 75 Days of Novel Coronavirus (SARS-CoV-2) Outbreak: Recent Advances, Prevention, and Treatment. Int J Environ Res Public Health. 2020;17(7):E2323. doi: 10.3390/ijerph17072323.

39. Roda WC, Varughese MB, Han D, Li MY. Why is it difficult to accurately predict the COVID-19 epidemic? Infect Dis Model. 2020;5:271-81. doi: 10.1016/j.idm.2020.03.001.

40. Panovska-Griffiths J. Can mathematical modelling solve the current Covid-19 crisis? BMC Public Health. 2020;20(1):551. doi: 10.1186/ s12889-020-08671-z.

41. Vargas-Uricoechea H. COVID-19 en Colombia e inmunidad de rebaño: ¿es momento de considerarla? Rev Colomb Endocr Diabetes Metab. 2020;7(1):57-9.

42. Ye Q Wang B, Mao J, Fu J, Shang S, Shu Q et al. Epidemiological analysis of COVID-19 and practical experience from China. J Med Virol. 2020. doi: $10.1002 / j m v .25813$.

43. Lewnard JA, Lo NC. Scientific and ethical basis for social-distancing interventions against COVID-19. Lancet Infect Dis. 2020. doi: 10.1016/S14733099(20)30190-0.

44. Logie CH, Turan JM. How Do We Balance Tensions Between COVID-19 Public Health Responses and Stigma Mitigation? Learning from HIV Research. AIDS Behav. 2020;1-4. doi: 10.1007/s10461-020-02856-8.

45. West R, Michie S, Rubin GJ, Amlôt R. Applying principles of behaviour change to reduce SARS-CoV-2 transmission. Nat Hum Behav. 2020;4(5):451-9. doi: 10.1038/s41562-020-0887-9.

46. Jarvis CI, Van Zandvoort K, Gimma A, Prem K; CMMID COVID-19 working group; Klepac P, et al. Quantifying the impact of physical distance measures on the transmission of COVID-19 in the UK. BMC Med. 2020;18(1):124 doi: 10.1186/s12916-020-01597-8.

47. Lai S, Ruktanonchai NW, Zhou L, Prosper O, Luo W, Floyd JR, et al. Effect of non-pharmaceutical interventions to contain COVID-19 in China. Nature. 2020. doi: 10.1038/s41586-020-2293-x.

48. Petersen E, Wasserman S, Lee SS, Go U, Holmes AH, Al Abri S, et al. COVID 19-We urgently need to start developing an exit strategy. Int J Infect Dis. 2020;96:233-9. doi: 10.1016/j.ijid.2020.04.035. 
49. Center for Infectious Disease Research and Policy. COVID-19: The CIDRAP Viewpoint [Internet]. [Citado 12 de mayo de 2020]. https://bit. ly/3cbDqNW.

50. Begley S. Three potential futures for Covid-19: recurring small outbreaks, a monster wave, or a persistent crisis [Internet]. [Citado 12 de mayo de 2020]. Disponible en: https://www.statnews.com/2020/05/01/threepotential-futures-for-covid-19/.
51. Glenn J. COVID-19 [Internet]. The Millennium Project. Global Studies \& Research. [Citado 12 de mayo de 2020]. Disponible en: http://www.millennium-project.org/covid-19/. 